\title{
Ocular phenotype and genetical analysis in patients with retinopathy of prematurity
}

\author{
Tianchang Tao ${ }^{1,2,3 \dagger}$, Xianfen Meng ${ }^{4 \dagger}$, Ningda Xu ${ }^{1,2,3}$, Jiarui $\mathrm{Li}^{1,2,3}$, Yong Cheng ${ }^{1,2,3}, \mathrm{Yi}_{\mathrm{C}}$ Chen $^{5^{*}}$ and \\ Lvzhen Huang ${ }^{1,2,3^{*}}$
}

\begin{abstract}
Background: Retinopathy of prematurity (ROP) is a multifactorial retinal disease, involving both environmental and genetic factors; The purpose of this study is to evaluate the clinical presentations and genetic variants in Chinese patients with ROP.

Methods: A total of 36 patients diagnosed with ROP were enrolled in this study, their medical and ophthalmic histories were obtained, and comprehensive clinical examinations were performed. Genomic DNA was isolated from peripheral blood of ROP patients, polymerase chain reaction and direct sequencing of the associated pathogenic genes (FZD4, TSPAN12, and NDP) were performed.

Results: All patients exhibited the clinical manifestations of ROP. No mutations were detected in the TSPAN12 and NDP genes in all patients; Interestingly, three novel missense mutations were identified in the FZD4 gene (p.A2P, p.L79M, and p.Y378C) in four patients, for a detection rate of $11.1 \%(4 / 36)$.
\end{abstract}

Conclusions: This study expands the genotypic spectrum of FZD4 gene in ROP patients, and our findings underscore the importance of obtaining molecular analyses and comprehensive health screening for this retinal disease.

Keywords: Retinopathy of prematurity, Frizzled-4, Wnt signaling pathway, Retinal vascular development

\section{Background}

Retinopathy of prematurity (ROP) is a complex ocular disease, characterized by abnormal retinal vascularization in premature infants; This disease is firstly described as retrolental fibroplasia in 1940s when a grayish white, opaque membrane behind the lens was observed in preterm infants [1]. ROP has become one of the leading causes of childhood blindness across the world, and the prevalence of ROP varies widely in different countries or areas [2-4], which should be associated with the regional

\footnotetext{
*Correspondence: chenyi88888@vip.sina.com; drlvzhenhuang@sina.com

†Tianchang Tao and Xianfen Meng contributed equally to this work.

${ }^{3}$ College of Optometry, Peking University Health Science Center, Beijing, China

${ }^{5}$ Department of Ophthalmology, China-Japan Friendship Hospital, Beijing, China

Full list of author information is available at the end of the article
}

economic development level and public health facilities. There is a two-phase hypothesis of ROP [5], in first phase, delayed retinal vascular development leads to a peripheral avascular area of the retina, and in second phase, angiogenesis can happen at the junction of avascularized and vascularized retina as a result of pathological compensatory mechanism, this process is considered to be attributed to the aberrant changes of oxygen tension and vascular endothelial growth factor (VEGF) level.

Although the exact mechanism of ROP remains unclear, many risk factors have been reported to induce ROP [6-9], such as low birth weight, young gestational age, supplemental oxygen, mechanical ventilation, apnea, anemia, and blood transfusion. In addition to those environmental factors, genetic mutations are also considered to get involved in ROP development, especially Wnt signaling factors: Frizzled-4 (FZD4), Tetraspanin-12 (TSPAN12), and Norrin (NDP), this signaling system original author(s) and the source, provide a link to the Creative Commons licence, and indicate if changes were made. The images or other third party material in this article are included in the article's Creative Commons licence, unless indicated otherwise in a credit line to the material. If material is not included in the article's Creative Commons licence and your intended use is not permitted by statutory regulation or exceeds the permitted use, you will need to obtain permission directly from the copyright holder. To view a copy of this licence, visit http://creativecommons.org/licenses/by/4.0/. The Creative Commons Public Domain Dedication waiver (http://creativeco mmons.org/publicdomain/zero/1.0/) applies to the data made available in this article, unless otherwise stated in a credit line to the data. 
plays and important role in fetal vascular formation of retina and inner ear $[10,11]$, and its functional disruption could be associated with the pathogenesis of some retinal vascular disorders like Coat's disease and familial exudative vitreoretinopathy (FEVR) $[12,13]$. Because of the phenotypic similarities between ROP and FEVR, some studies indicate the possibility of involvement of Wnt signaling genes mutations in the occurrence of ROP [14-16]. Thus, further studies are required to investigate the correlation between Wnt signaling abnormality and aberrant retinal vascular development in ROP patients.

Here, our study investigated 36 patients diagnosed with ROP, we obtained comprehensive histories of their illnesses, and then evaluated the effect of pathogenic genes variants on their ocular features.

\section{Methods \\ Patients}

Thirty-six ROP patients from thirty-three unrelated families were enrolled in this study, the informed consent was obtained from all guardians on behalf of the participants. Our study conformed to the tenets of the Declaration of Helsinki. The procedure was approved by the ethics committee of Peking University People's Hospital.

Data for gender, gestational age (GA) at birth, birth weight (BW) were collected, and complete medical histories were consulted if available. The results of ophthalmic examinations, such as indirect ophthalmoscopy, slit-lamp biomicroscopy, and color fundus photography, were collected where available. The clinical tests obtained in our study were performed before treatment. The stage of ROP was assessed for each patient following the International Classification of Retinopathy of Prematurity [17]: Stage 0 presents only immature retinal vasculature; Stage 1 presents a demarcation line between vascularized and avascular retina; Stage 2 presents a ridge characterized by the demarcation line with height, width, and volume; Stage 3 presents the extraretinal fibrovascular proliferation or neovascularization extending from the ridge into the vitreous; Stage 4 is divided into extrafoveal (4A) or foveal (4B) partial retinal detachment; Stage 5 presents the total retinal detachment.

\section{Molecular analysis}

Genomic DNA was extracted from peripheral blood of ROP patients using an Agilent SureSelect Target Enrichment System Kit (Agilent, USA). Polymerase chain reaction (PCR) was performed using Goldstar Taq MasterMix (Cwbio, PRC) to amplify the exons of the FZD4, TSPAN12, and NDP genes. The samples were sequenced and analyzed by NEXTSEQ500 (Illumina, USA) as described previously [18].
The possible pathogenicity of novel missense mutations would be evaluated by using SIFT (http://sift.jcvi.org/), PolyPhen-2 (http://genetics.bwh.harvard.edu/pph2/), and Mutation Taster (http://www.mutationtaster.org) prediction software and via evolutionary conservation analysis. The minor allele frequencies (MAF) of variants in participants were checked using Exome Aggregation Consortium database (http://exac.broadinstitute.org/ faq) and Genome Aggregation database (http://gnomadsg.org/); All mutations were also evaluated regarding pathogenicity according to the standards and guidelines of American College of Medical Genetics and Genomics (ACMG) [19].

\section{Results}

Demographic data for the 36 participants are presented in Table 1. Twenty-three patients were boys (63.9\%) and thirteen were girls (36.1\%). The average gestational age was 30 weeks with a range from 27 to 36 weeks, and the average birth weight was $1300 \mathrm{~g}$ with a range from 890 to $2700 \mathrm{~g}$; Thirty-three patients $(91.7 \%)$ presented the ocular manifestation of classic ROP, and the aggressive posterior ROP (AP-ROP) was found in three patients (8.3\%); Among the 36 cases, three patients $(8.3 \%)$ were at stage 0 , one patient $(2.8 \%)$ was at stage 1 , one patient $(2.8 \%)$ was at stage 2, 20 patients (57.4\%) were classified as stage 3, two patients $(5.6 \%)$ were at stage $4 \mathrm{~A}$, two patients $(5.6 \%)$ were at stage $4 \mathrm{~B}$, and the other seven patients (19.3\%) were not determined. Twenty-eight patients (77.8\%) underwent intravitreal anti-VEGF agent or laser photocoagulation after screening and diagnosis.

FZD4 gene mutations were identified in four of the 36 ROP patients enrolled in our study, including three

Table 1 Demographic data for 36 patients with retinopathy of prematurity

\begin{tabular}{ll}
\hline Gestational age & $27-36$ (mean 30) weeks \\
Birth weight & $890-2700$ (mean 1300) grams \\
Sex & Male: $23(63.9 \%)$ \\
& Female: $13(36.1 \%)$ \\
Type of ROP & AP-ROP: $3(8.3 \%)$ \\
& Classic ROP: 33 (91.7\%) \\
Stage of ROPa & Stage 0: $3(8.3 \%)$ \\
& Stage 1: $1(2.8 \%)$ \\
& Stage 2: $1(2.8 \%)$ \\
& Stage 3: $20(55.6 \%)$ \\
& Stage 4A: $2(5.6 \%)$ \\
& Stage 4B: $2(5.6 \%)$ \\
Undetermined: $7(19.3 \%)$
\end{tabular}

${ }^{a}$ The severity of patients was determined by the highest stage of ROP in either eye 


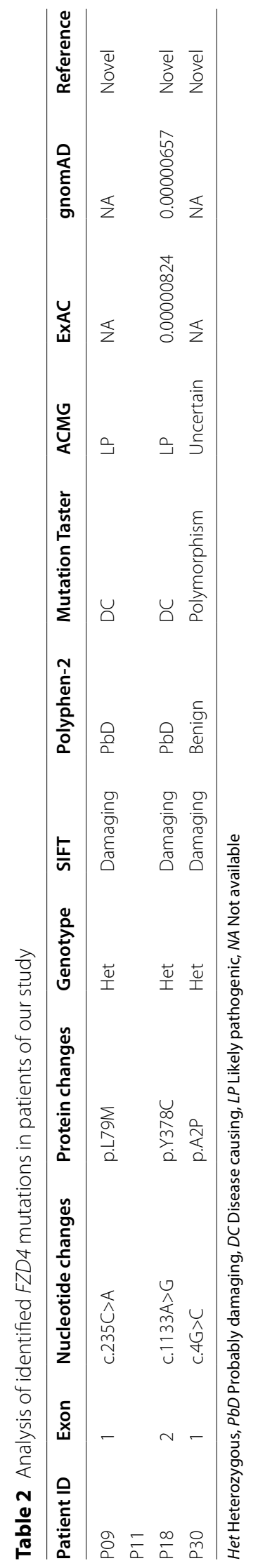


novel missense mutations (Table 2): p.A2P, p.L79M, and p.Y378C. No variants were found for TSPAN12 and NDP genes in this study.

The clinical findings of the four patients who carried FZD4 mutations are shown in Table 3. The variant p.L79M was detected in two patients with classic ROP. One patient was a boy (P09) who was born at a gestational age of 28 weeks with a birthweight of $1250 \mathrm{~g}$; The retinopathy progressed to stage 3 in both eyes (Fig. 1a); Intravitreal ranibizumab (IVR) therapy was performed on both eyes at 10 weeks after birth. The other patient was a boy (P11) who was born at a gestational age of 33 weeks with a birth weight of $2400 \mathrm{~g}$; The retinopathy developed to stage 4A bilaterally (Fig. 1b), and IVR treatment was performed on both eyes at 7 weeks after birth.

The p.Y378C mutation was found in one boy with classic ROP (P18). He was born at a gestational age of 30 weeks with a birthweight of $1150 \mathrm{~g}$, and his fundus photography presented neovascular or proliferative alterations in both eyes (Fig. 1c), which are associated with stage 3 retinopathy; Bilateral IVR therapy was performed at 9 weeks after birth. The p.A2P mutation was detected in a girl (P30), she was born at a gestational age of 32 weeks with a birth weight of $1950 \mathrm{~g}$, and her fundus photography showed immature retinal vascularization (stage 0) in both eyes (Fig. 1d).

The two mutations p.L79M and p.Y378C are predicted to be damaging, probably damaging, and disease causing according to SIFT, PolyPhen-2, and Mutation Taster, and p.A2P is predicted to be damaging, benign, and polymorphism; In addition, p.A2P and p.L79M are never detected in ExAC and gnomAD, and p.Y378C is shown to be rare in the two databases $(0.00000824$ in ExAC, and 0.00000657 in gnomAD); According to the standards established by the ACMG for the classification of variants, p.L79M and p.Y378C are categorized as likely pathogenic, and p.A2P is classified as a variant with uncertain significance (Table 2). Additionally, Codon 2 is located in the signal sequence portion of FZD4, codon 79 lies upstream of cysteine-rich domain (CRD), and codon 378 is within the seven transmembrane domains (TMDs). Codon 79 and 378 are highly conserved in vertebrates, and codon 2 is conserved in all species except chicken, monkey, elephant and frog (Fig. 2). The CRD belongs to the extracellular region of FZD4 protein, and is found to be responsible for Norrin/ $\beta$-catenin signaling pathway, as Norrin specifically binds to the CRD of FZD4 but not to CRDs of other 14 mammalian Frizzled or secreted Frizzled-related proteins [20], the mutational effects on CRD have been verified by altered cellular processing, plasma membrane targeting, interaction with Norrin ligand, and the ability to activate the signaling pathway [21]. The TMD is conserved among all the putative paralogs of the frizzled receptor family [22], and contains seven transmembrane $\alpha$-helices [23]; The carboxyl-terminal region possesses a conserved K-T/S-XXX-W PDZ-binding motif that locates immediately after the hydrophobic TMD, this motif is necessary for canonical Wnt pathway initiation and Disheveled (Dvl) protein phosphorylation, and interaction of FZD4 with Dvl completely diminishes while the motif encountering any mutation [24]. The aminoterminal membrane localizing signal peptide sequence is rich in hydrophobic residues, and is known to translocate FZD4 to the plasma membrane, mutations that occur in this region might result in impaired stability of FZD4 protein and its mistranslocation subsequently [25].

\section{Discussion}

ROP is known as a multifactorial disorder, gestational age, birth weight and supplemental oxygen are common risk factors. FEVR is an inherited retinal disorder characterized by incomplete retinal vascular development and secondary retinal changes, and has been reported to be associated with Wnt signaling genes (FZD4, TSPAN12, and $N D P$ ) polymorphism [26, 27]. As both diseases share similar clinical characteristics, the variants in these FEVR-causing genes are also considered to contribute to ROP pathogenesis. Here, our study showed that four of 36 ROP patients harbored three novel variants p.A2P, p.L79M, and p.Y378C in FZD4 gene, no such sequence alterations were detected in TSPAN12 and NDP genes.

FZD4 is located on chromosome 11q14.2, and encodes a 537 amino acid protein, which belongs to the frizzled receptor family. It consists of a $\mathrm{N}$-terminal extracellular cysteine-rich domain, seven transmembrane domains, extracellular/intracellular loops, and a C-terminal

Table 3 Clinical characteristics of four ROP patients carrying FZD4 mutations

\begin{tabular}{llllll}
\hline Patient ID & Gender & Gestational age & Birth weight & Stage of ROP & Treatment \\
\hline P09 & Male & $28 \mathrm{w}$ & $1250 \mathrm{~g}$ & $3(\mathrm{OU})$ & IVR (OU) at 10 w after birth \\
P11 & Male & $33 \mathrm{w}$ & $2400 \mathrm{~g}$ & $4 \mathrm{~A}(\mathrm{OU})$ & IVR (OU) at 7 w after birth \\
P18 & Male & $30 \mathrm{w}$ & $1150 \mathrm{~g}$ & $3(\mathrm{OU})$ & IVR (OU) at 9 w after birth \\
P30 & Female & $32 \mathrm{w}$ & $1950 \mathrm{~g}$ & $0(\mathrm{OU})$ & - \\
\hline
\end{tabular}

$w$ weeks, $g$ grams, IVR intravitreal ranibizumab 

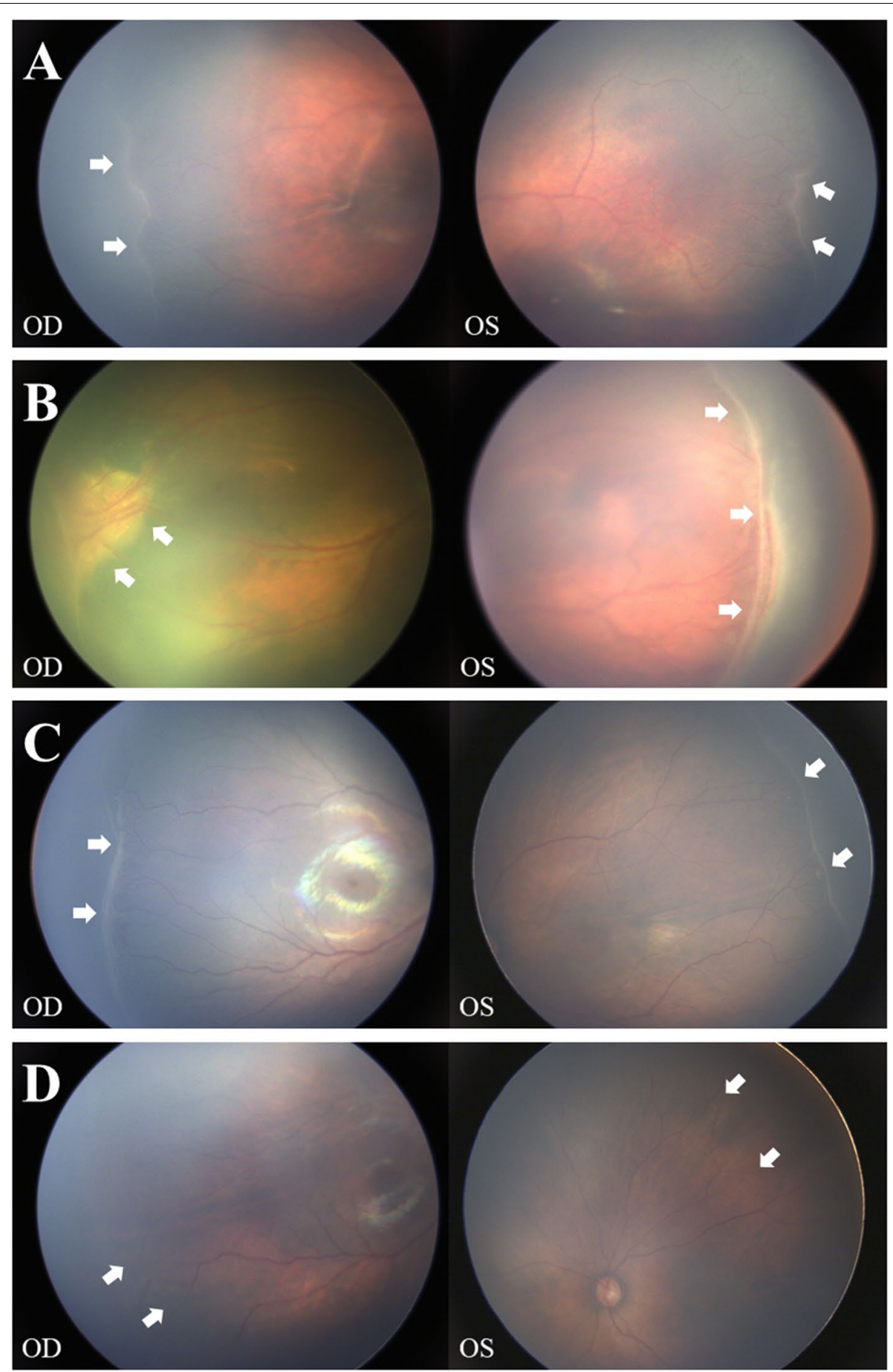

Fig. 1 The color fundus photograph (CFP) of four ROP patients carrying mutations in FZD4 gene. a The CFP of P09 demonstrates stage 3 of ROP, white arrows indicate the temporal irregular sprouts of vascularization at the ridge between vascular and avascular area. $\mathbf{b}$ The CFP of P11 demonstrates stage 4A of ROP, white arrows indicate the temporal partial retinal detachment. c The CFP of P18 demonstrates the temporal neovascular or proliferative alterations, which are related to stage 3 retinopathy and marked by white arrows. $\mathbf{d}$ The CFP of P30 demonstrates stage 0 of ROP, white arrows indicate the progressive tapering of retinal vessels 
FZD4 protein region

signal sequence

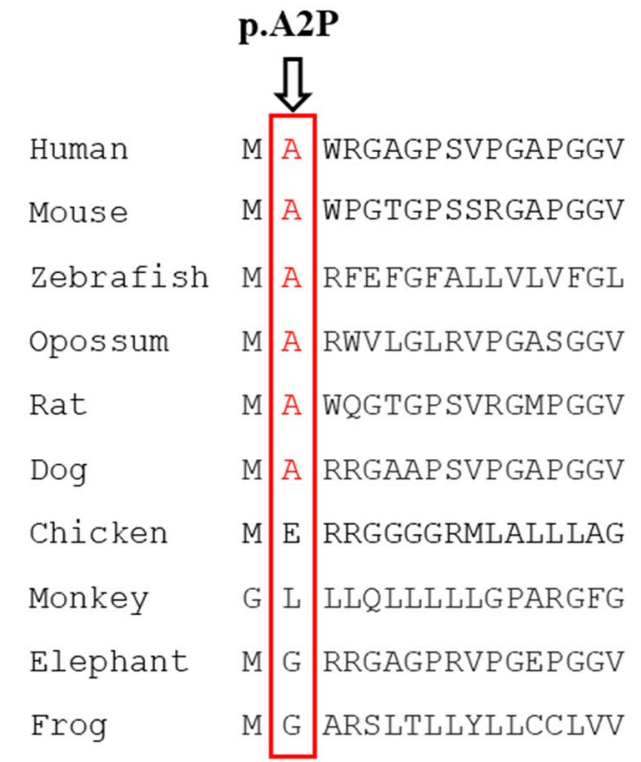

cysteine-rich domain

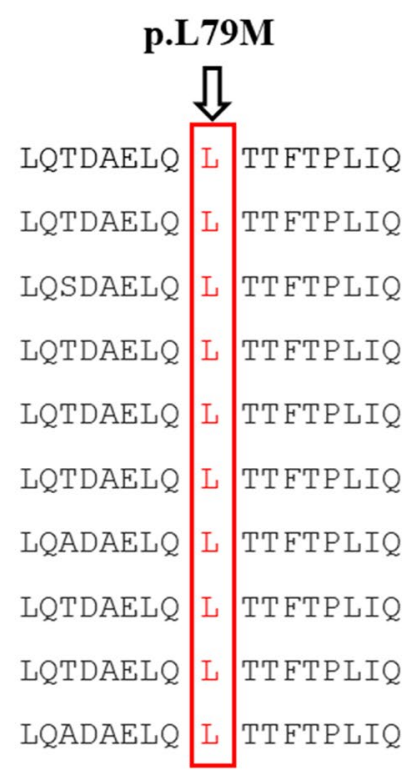

transmembrane domain

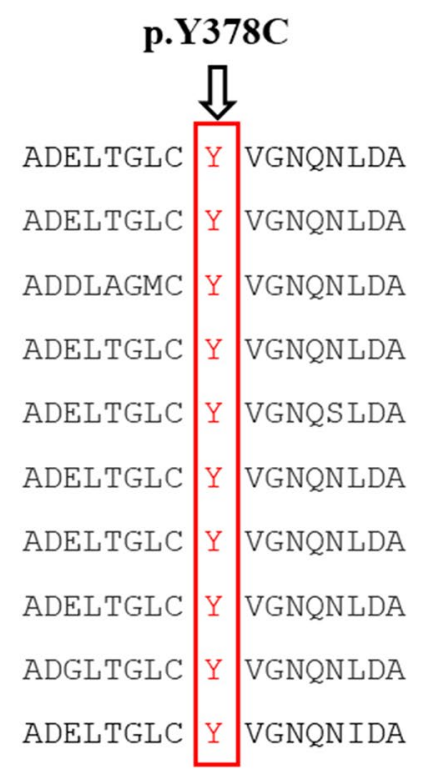

Fig. 2 Sequence alignment of the FZD4 protein. Amino acids are shown by one letter code and red rectangles indicate the corresponding codons to FZD4 variants found in our study, including codons A2, L79, and Y378

intracellular domain [28]. Frizzled-4 plays the role of receptor to bind specific ligands, which is essential for initiating different signaling pathways, such as canonical Wnt/ $\beta$-catenin pathway, planar cell polarity pathway, and Wnt $/ \mathrm{Ca}^{2+}$ pathway, this signaling pathway impairments could cause defective tissue homeostasis or cell proliferation in retina [29].

The p.L79M mutation in P09 and P11 replaces the nonpolar leucine residue with the neutral and uncharged methionine residue; Furthermore, methionine is large, whereas leucine is small. This single-base substitution might cause an impact on CRD of FZD4, and lead to disrupted interaction between CRD and its ligands, further resulting in Wnt signaling dysfunction and retinal vascular alternations; All the structural or functional changes, combined with the absence of the FZD4 variant in databases, indicate its pathogenicity in ROP. Furthermore, the gestational age and birth weight of P11 are relatively higher than that of other three patients with FZD4 mutations, and his clinical features are similar to that of Li et al. [16], who reported seven atypical ROP patients presenting significantly greater gestational age and birth weight compared with typical ROP patients, so the phenotype of P11 might be attributed to the type of atypical ROP. It is noteworthy that P09 and P11 underwent different degrees of prematurity or other systemic abnormalities, the phenotypic severity in two patients carrying the same variant appeared to be inconsistent, suggesting that both genetic and environmental factors could affect the phenotypic heterogeneity in ROP.

In P18, variant p.Y378C is located in the seven transmembrane domains, tyrosine 378 belongs to the transmembrane ligand pocket of FZD4 [30], which is highly conserved in all species (Fig. 2); This mutation replaces the tyrosine residue with the smaller cysteine residue, it could impair the function of TMD and cause alterations in Wnt signaling pathway activation. Besides, the p.A2P mutated in P30 is in the signal sequence of $F Z D 4$, and could affect the process of FZD4 translocation to the plasma membrane; In this mutation, the non-polar alanine residue is replaced by a larger proline residue with the same properties, proline is an atypical amino acid with the characteristic of rigidity, which is crucial for the proper conformation of protein. Interestingly, P30 exhibited a milder phenotype compared with other three FZD4-mutated patients, this might be explained that the rigidity of proline did not affecting the important function of FZD4 protein completely, indicating the severity of mutational effect of FZD4 gene could result in different ROP phenotypes. This finding is similar to that of Hiroyuki et al. [15], who suggested phenotypic severities being related to genotypic severities in patients with ROP. 


\section{Conclusion}

In this study, disease-causing mutations were not found in nearly $90 \%$ of participants in our study. No significant variants were detected in TSPAN12 and NDP genes. Due to the limited number of ROP cases enrolled in this study, the correlation between phenotype and genotype in ROP patients was not entirely elucidated; In conclusion, our findings showed three novel missense mutations (p.A2P, p.L79M, and p.Y378C) of the FZD4 gene, and broadened the mutational spectrum of ROP, implying the potential unique role of FZD4 in ROP pathogenesis; Although ROP seems to be a complex disorder involving both environmental and genetic factors, this study could provide useful information for better exploring the mechanism of this retinal vascular disease, thus highlighting the necessity of performing a comprehensive clinical and genetic screening to make progress in the diagnosis and treatment of ROP.

\section{Supplementary Information}

The online version contains supplementary material available at https://doi. org/10.1186/s12886-022-02252-x.

Additional file 1: Table S1. Clinical findings of thirty-six patients with ROP.

\section{Acknowledgements}

The authors thank all patients and their families for their participation in this study.

\section{Authors' contributions}

YC and LZH are co-corresponding authors, who contributed to project design and the revision of the manuscript. TCT and XFM are co-first authors, who contributed to data analysis, and drafted the manuscript. NDX, JRL, and YC contributed to data collection and manuscript discussion. All authors read and approved the final manuscript.

\section{Funding}

This study was supported by the National Natural Science Foundation of China Grant $(81470649,81670870)$, the National Key Research and Development Program of China (2020YFC2008200), and the Science and Technology Innovation Project of Chinese Academy of Medical Sciences (2019-RC-HL-019).

\section{Availability of data and materials}

The data that support the findings of this study are available upon reasonable request from the corresponding author.

\section{Declarations}

Ethics approval and consent to participate

The procedure was approved by the ethics committee of Peking University People's Hospital. The informed consent was obtained from all guardians on behalf of the participants and our study conformed to the tenets of the Declaration of Helsinki.

\section{Consent for publication}

The informed consent for publication was obtained from all guardians on behalf of the participants.

\section{Competing interests}

The authors declare that they have no competing interests.

\section{Author details}

${ }^{1}$ Department of Ophthalmology, Peking University People's Hospital, Eye Diseases and Optometry Institute, Beijing, China. ${ }^{2}$ Beijing Key Laboratory of Diagnosis and Therapy of Retinal and Choroid Diseases, Beijing, China. ${ }^{3} \mathrm{Col}-$ lege of Optometry, Peking University Health Science Center, Beijing, China. ${ }^{4}$ Department of Ophthalmology, Beijing Huaxin Hospital, The First Hospital of Tsinghua University, Beijing, China. ${ }^{5}$ Department of Ophthalmology, ChinaJapan Friendship Hospital, Beijing, China.

Received: 25 May 2021 Accepted: 6 January 2022

Published online: 12 January 2022

\section{References}

1. Terry TL. Extreme prematurity and fibroblastic overgrowth of persistent vascular sheath behind each crystalline Lens: I. Preliminary report. Am J Ophthalmol. 1942;192(25):203-4.

2. Vinekar A, Dogra MR, Sangtam T, Narang A, Gupta A. Retinopathy of prematurity in Asian Indian babies weighing greater than 1250 grams at birth: ten year data from a tertiary care center in a developing country. Indian J Ophthalmol. 2007;55(5):331-6.

3. Austeng D, Källen KB, Ewald UW, Jakobsson PG, Holmström GE. Incidence of retinopathy of prematurity in infants born before 27 weeks' gestation in Sweden. Arch Ophthalmol. 2009;127(10):1315-9.

4. Darlow BA, Hutchinson JL, Henderson-Smart DJ, Donoghue DA, Simpson $J M$, Evans NJ. Prenatal risk factors for severe retinopathy of prematurity among very preterm infants of the Australian and New Zealand neonatal network. Pediatrics. 2005;115(4):990-6.

5. Hartnett ME, Penn JS. Mechanisms and management of retinopathy of prematurity. N Engl J Med. 2012;367(26):2515-26.

6. Karna P, Muttineni J, Angell L, Karmaus W. Retinopathy of prematurity and risk factors: a prospective cohort study. BMC Pediatr. 2005;5(1):18.

7. Giannantonio C, Papacci P, Cota F, Vento G, Tesfagabir MG, Purcaro V, et al. Analysis of risk factors for progression to treatment-requiring ROP in a single neonatal intensive care unit: is the exposure time relevant? J Matern Fetal Neonatal Med. 2012;25(5):471-7.

8. Solans Pérez de Larraya AM, Ortega Molina JM, Uberos Fernández J, González Ramírez AR, García Serrano JL. Speed of retinal vascularization in retinopathy of prematurity: risk and protective factors. Biomed Res Int. 2019;2019:2721578.

9. Sanghi G, Dogra MR, Dutta S, Katoch D, Gupta A. Intersibling variability of retinopathy of prematurity in twins and its risk factors. Int Ophthalmol. 2012;32(2):113-7.

10. Xu Q, Wang Y, Dabdoub A, Smallwood PM, Williams J, Woods C, et al. Vascular development in the retina and inner ear: control by Norrin and Frizzled-4, a high-affinity ligand-receptor pair. Cell. 2004;116(6):883-95.

11. Zhou Y, Wang Y, Tischfield M, Williams J, Smallwood PM, Rattner A, et al. Canonical WNT signaling components in vascular development and barrier formation. J Clin Invest. 2014;124(9):3825-46.

12. Seo SH, Yu YS, Park SW, Kim JH, Kim HK, Cho SI, et al. Molecular characterization of FZD4, LRP5, and TSPAN12 in familial exudative Vitreoretinopathy. Invest Ophthalmol Vis Sci. 2015;56(9):5143-51.

13. Robitaille JM, Zheng B, Wallace K, Beis MJ, Tatlidil C, Yang J, et al. The role of Frizzled-4 mutations in familial exudative vitreoretinopathy and coats disease. Br J Ophthalmol. 2011;95(4):574-9.

14. Ells A, Guernsey DL, Wallace K, Zheng B, Vincer M, Allen A, et al. Severe retinopathy of prematurity associated with FZD4 mutations. Ophthalmic Genet. 2010;31(1):37-43.

15. Kondo H, Kusaka S, Yoshinaga A, Uchio E, Tawara A, Tahira T. Genetic variants of FZD4 and LRP5 genes in patients with advanced retinopathy of prematurity. Mol Vis. 2013;19:476-85.

16. Li Y, Li J, Zhang X, Peng J, Li J, Zhao P. Identification of gene mutations in atypical retinopathy of prematurity cases. J Ophthalmol. 2020;2020:4212158.

17. Chiang MF, Quinn GE, Fielder AR, Ostmo SR, Paul Chan RV, Berrocal A, et al. International classification of retinopathy of prematurity, Third Edition. Ophthalmology. 2021;128(10):e51-68.

18. Gao T, Tian C, Hu Q, Liu Z, Zou J, Huang L, et al. Clinical and mutation analysis of patients with best Vitelliform macular dystrophy or autosomal 
recessive Bestrophinopathy in Chinese population. Biomed Res Int. 2018;2018:4582816.

19. Richards S, Aziz N, Bale S, Bick D, Das S, Gastier-Foster J, et al. Standards and guidelines for the interpretation of sequence variants: a joint consensus recommendation of the American College of Medical Genetics and Genomics and the Association for Molecular Pathology. Genet Med. 2015;17(5):405-24.

20. Smallwood PM, Williams J, Xu Q, Leahy DJ, Nathans J. Mutational analysis of Norrin-Frizzled4 recognition. J Biol Chem. 2007:282(6):4057-68.

21. Zhang K, Harada Y, Wei X, Shukla D, Rajendran A, Tawansy K, et al. An essential role of the cysteine-rich domain of FZD4 in Norrin/ Wnt signaling and familial exudative vitreoretinopathy. J Biol Chem. 2011:286(12):10210-5.

22. Seemab S, Pervaiz N, Zehra R, Anwar S, Bao Y, Abbasi AA. Molecular evolutionary and structural analysis of familial exudative vitreoretinopathy associated FZD4 gene. BMC Evol Biol. 2019;19(1):72.

23. Ramasarma T. Transmembrane domains participate in functions of integral membrane proteins. Indian J Biochem Biophys. 1996;33(1):20-9.

24. Wong HC, Bourdelas A, Krauss A, Lee HJ, Shao Y, Wu D, et al. Direct binding of the PDZ domain of Dishevelled to a conserved internal sequence in the C-terminal region of frizzled. Mol Cell. 2003;12(5):1251-60.

25. Drenser KA, Dailey W, Vinekar A, Dalal K, Capone A Jr, Trese MT. Clinical presentation and genetic correlation of patients with mutations affecting the FZD4 gene. Arch Ophthalmol. 2009;127(12):1649-54.

26. Li JK, Li Y, Zhang X, Chen CL, Rao YQ, Fei P, et al. Spectrum of variants in 389 Chinese Probands with familial exudative Vitreoretinopathy. Invest Ophthalmol Vis Sci. 2018;59(13):5368-81.

27. Wang Z, Chen C, Sun L, Zhang A, Liu C, Huang L, et al. Symmetry of folds in FEVR: a genotype-phenotype correlation study. Exp Eye Res. 2019;186:107720.

28. Xiao H, Tong Y, Zhu Y, Peng M. Familial exudative Vitreoretinopathyrelated disease-causing genes and Norrin/ $\beta$-catenin signal pathway: structure, function, and mutation spectrums. J Ophthalmol. 2019;2019:5782536.

29. Caceres L, Prykhozhij SV, Cairns E, Gjerde H, Duff NM, Collett K, et al. Frizzled 4 regulates ventral blood vessel remodeling in the zebrafish retina. Dev Dyn. 2019;248(12):1243-56.

30. Yang $S, W u Y, X u T H$, de Waal PW, He Y, Pu M, et al. Crystal structure of the frizzled 4 receptor in a ligand-free state. Nature. 2018;560(7720):666-70.

\section{Publisher's Note}

Springer Nature remains neutral with regard to jurisdictional claims in published maps and institutional affiliations.

Ready to submit your research? Choose BMC and benefit from:

- fast, convenient online submission

- thorough peer review by experienced researchers in your field

- rapid publication on acceptance

- support for research data, including large and complex data types

- gold Open Access which fosters wider collaboration and increased citations

- maximum visibility for your research: over $100 \mathrm{M}$ website views per year

At BMC, research is always in progress.

Learn more biomedcentral.com/submissions 